\title{
The Industrial Development Path Research of Chinese Herbal Medicine From the Perspective of Whole Industry Chain
}

\author{
Xin $\mathrm{Su}^{1, \mathrm{a}}$, Chunlan $\mathrm{Mou}^{2, \mathrm{~b}}$, Xiaohui Wang ${ }^{2, *}$ \\ ${ }^{1}$ School of Business Administration Shandong University of Finance and Economic \\ No.7366, East Erhuan Road, Jinan, 250014 China \\ ${ }^{2}$ School of Business Administration Shandong University of Finance and Economic \\ No.7366, East Erhuan Road, Jinan, 250014 China \\ agj_sx@126.com, ${ }^{\text {b}}$ zuiaidul@163.com, * lanse703@163.com \\ (*means corresponding author)
}

Keywords: Chinese herbal medicine; Whole industry chain perspective; Value chain; Development path

\begin{abstract}
The industrialization development of Chinese herbal medicine is a systematic project involving many aspects. With the government support and the unique advantages of traditional Chinese medicine in the field of health care and prevention, the demand for Chinese herbal medicine is increasing day by day. The whole industry chain management is one of the strategic factors for the development of Chinese herbal medicine industrialization from factor driven, investment driven to innovation driven. This article, through the analysis of related problems, optimized constructs the Chinese herbal medicine industry development path from the perspective of the whole industry chain, and in-depth analyzes the implementation process of the strategy, which is very significant to enhance the core competitiveness of Chinese herbal medicine industry.
\end{abstract}

\section{Introduction}

With the unique advantage in the field of health, prevention and the government support, the demand of Chinese herbal medicine is increasing. At the same time , the supply of wild medicinal herbs is decreasing. Therefore, the need for artificial cultivation of Chinese herbal medicine is urgent. The industrialization development of Chinese herbal medicine is not only the demand of social health, but also the reform of the supply side structure of the country, it is the only way to promote the transformation from old to new kinetic energy. Through integrating the one two three industry, "market oriented" as the guide, "science and technology" as the support, "new business entities" as the carrier, "scientific management" ${ }^{[1]}$ as means to build a new model of the whole industry chain innovation platform construction. There is little research on Chinese herbal medicine industry chain abroad, and there is no systematic theory. Therefore, more research is carried out on the present situation of Chinese herbal medicine industry.

\section{Problem analysis}

\subsection{Industry chain is unbalanced}

Chinese herbal medicine planting area is relatively large, high yield, but its processing level is low, showing the mismatch phenomenon ${ }^{[2]}$. Most the leading enterprises and cooperatives are mainly concentrated on the planting, cleaning, slicing and other early processing. In general the amount of resources is large , but the value is small, the level of industrialization is low, the industrial chain is low-end, and didn't form a complete industrial chain. The establishment of Chinese herbal medicine industry from production to consumption with a chain system of quality assurance is particularly important, but the lack of tracking mechanism of purity refining technology 
and production, manufacturing and sales of Chinese herbal medicine is present. Logistics level is relatively lagging which hinder the development of the whole industry chain ${ }^{[3]}$

\subsection{Industry chain value potential has not been excavated}

How to do more high-end traditional industries, increase value-added products, enhance their profit margins, has not yet been excavated. And also it has not yet been fully exploited about how to integrate resources, create high-end brand extension to the consumer terminal on the basis of high quality raw materials, so the brand value can be reflected in the value chain node, to realize the value added of industrial chain. And then we can develop a comprehensive industrial value chain which integrates planting, processing, tourism and health services, and forms a characteristic industrial cluster. The depth research and value mining of genuine Chinese herbal medicine is not enough. Most enterprises do not have long-term plans for R \& D, lack of core technology and innovative products with independent intellectual property rights, and can only imitate, OEM, and can not stand at the forefront of industrial development.

\section{Route optimization}

In the study of the industry chain, many domestic and foreign scholars mainly from the micro level to research the multi dimension of industry chain, such as supply chain, information chain and value chain. Michael Potter thinks the value of raw materials in the process of the value chain is actually implemented by a correlation in different sectors of the industry chain. This paper is based on the value chain perspective of Michael Potter, to research a series of value-added process about Chinese herbal medicine, from breeding and production material purchasing before planting to provide after-sales service until final sales are completed, as illustrated in Figure 1. This process is an effective path for the industrialization of Chinese herbal medicine in the perspective of the whole industry chain.

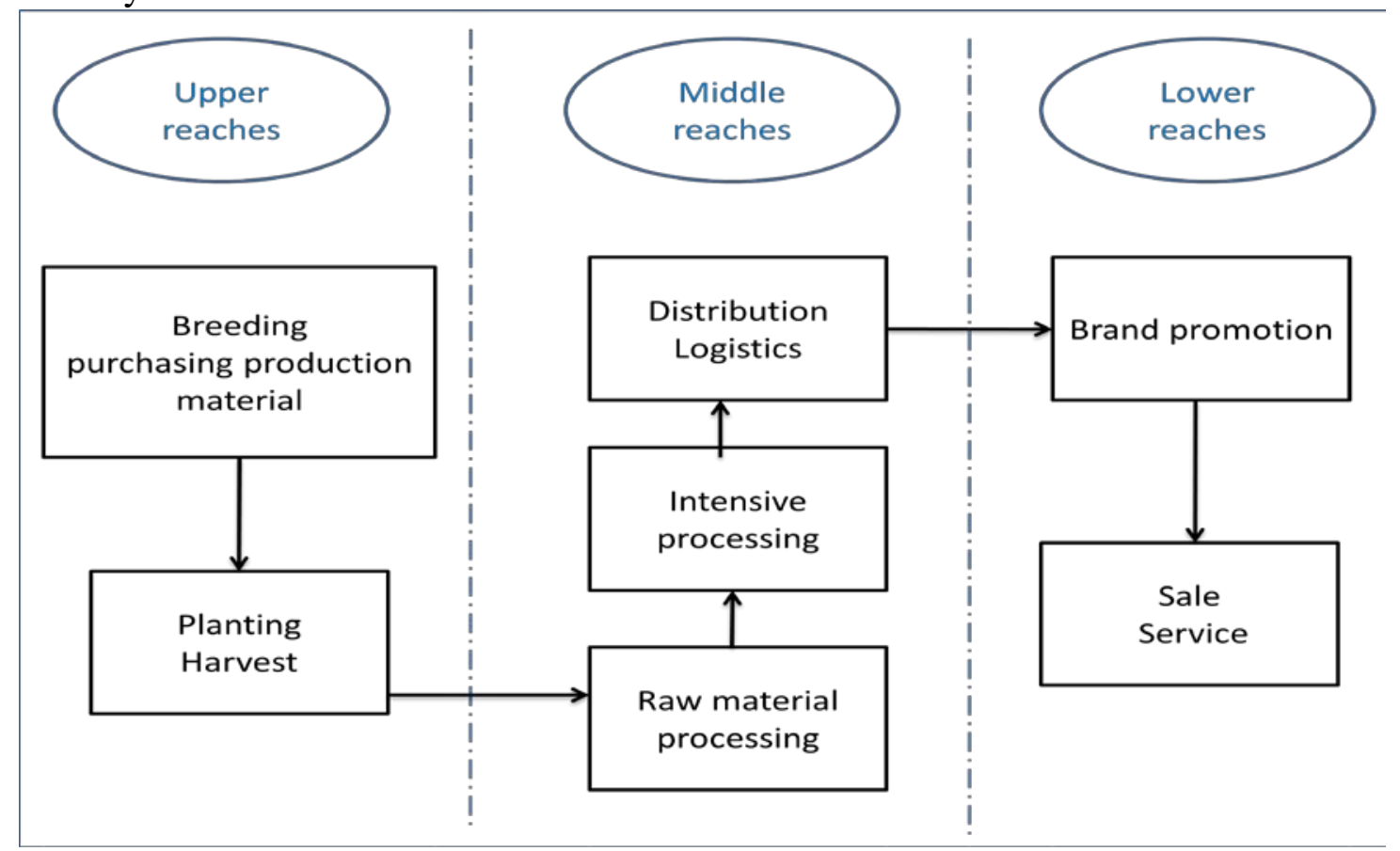

Figure 1: Chinese herbal medicine industry chain system

In terms of its composition, the industrial chain is a concept of four dimensions, including value chain, enterprise chain, supply chain and spatial chain. The four dimensions formed the industrial chain in the process of balanced docking. This "docking mechanism" is the internal model of industrial chain (Figure two), and as an objective law exist, it is like a "invisible hand" to regulate the formation of the industrial chain. 


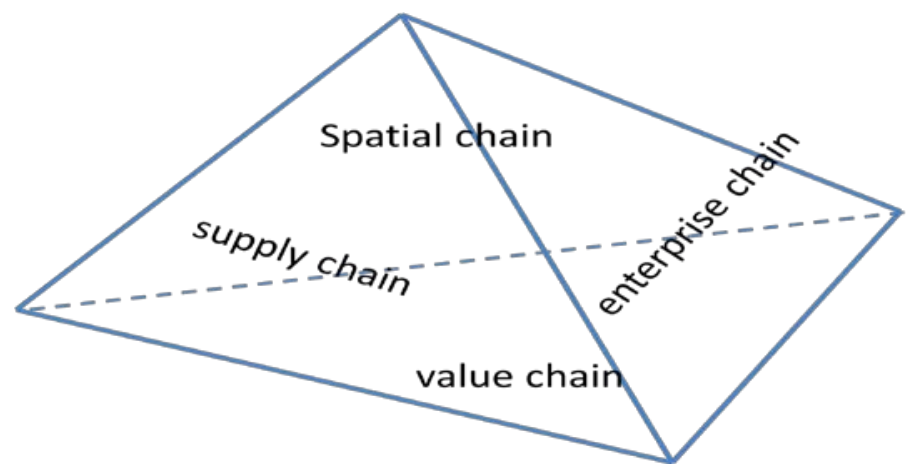

Figure 2: Concept map of industrial chain

The development of any industry cannot do without driving related industries. The industrial chain is generally divided into three parts: upper reaches, middle reaches and lower reaches. Only the middle reaches and lower reaches patency, the water upper reaches flows to lower reaches,.Chinese herbal medicine planting belongs to the upstream industry, but our country lacks middle and lower reaches related industries to cooperate with it. Therefore, we should explore, innovate and cultivate a complete chain of Chinese herbal medicine industry, that is, to optimize the development path of Chinese herbal medicine industry from the perspective of the whole industry chain.

\section{Path implementation}

\subsection{Strategic coordination}

The "whole industry chain" model refers to the complete industrial chain system consisting of planting and purchasing, raw materials / processing, distribution / logistics, brand promotion, sales and service, etc. from the field to the table. The new competitiveness kinetic energy of Chinese herbal medicine industry must come from the industry itself finally, by creating the whole industry chain mode, impenetrating the industry sectors of purchasing production materials, planting, processing, selling and brand building, fusing the new technology and new ideas about planting and processing technology, internet and electronic commerce, market economy and enterprise management, and finally, the "1+1>2" effect will be formed, and a new pattern of innovation and difference will be formed.

\subsection{Three chain reconstruction}

The future development of Chinese herbal medicine industry has demonstrated from a single industry to a multi industry and multi-functional comprehensive format, that is, the Chinese herbal medicine industry obtains new development momentum through the "three chain reconstruction". The so-called "three chain reconstruction" means the extension of industry chain, value chain increment and supply chain penetration. Industrial chain extension refers to the further refining and the commercialization of Chinese herbal medicine until it meets the needs of consumers. Through the survey we found that the region with high Chinese herbal medicine planting quality, its price and reputation in the market is relatively flat. Therefore, fine management, further high-end brand is imperative. From internalizing the characteristics of green, organic and ecological sustainability in the product itself by formulating industry standards and technical standards system, industry chain can realize its value added. The same quality primary products of different production base can enter the next value node through the same channels by means of integration and information sharing in supply chain. This can achieve brand effect, the lowest cost of the entire value chain, and achieve real cross-border integration.

\subsection{Cultivate new subjects}

Through the cultivation of leading enterprises of Chinese herbal medicine, large planting and 
processing enterprises, Chinese herbal medicine cooperation organizations, Chinese herbal medicine family farms and other forms of new industry management, the Chinese herbal medicine industry will be upgraded. The upgrade requires market, green development oriented, strengthen brand building, and it is difficult to achieve obviously rely on the traditional "peasant" business. The new business entities, whose ability to receive new ideas, new techniques and new methods is strong, have the strong will and ability to carry out brand building and promotion. At the same time, we must cultivate new vocational farmers.

\subsection{Strengthen the policy and regulations environment support system}

Chinese herbal medicine industry upgrading is mainly to guide the Chinese herbal medicine industry operators actively upgrade, it is essential to create a favorable policy environment supporting system, which may involve support policies and laws of finance, infrastructure, personnel, information, market and technology.

\subsection{Make full use of the Internet and exploit the advantages of e-commerce}

Electronic commerce is a kind of efficient, fast, low cost of trade.The high efficient operation is its development direction. The efficiency of e-commerce is very effective in promoting the development of the whole industry chain of Chinese herbal medicine.

\section{Conclusion}

The development of Chinese herbal medicine industry is still in the initial stage, and the development trend is its whole industry chain perspective and depth direction. In this paper, the optimized route of Chinese herbal medicine industry is constructed from the perspective of the whole industry chain, and a concrete and feasible strategy is put forward to realize this path. It is a good guideline for the upgrading and development of Chinese herbal medicine industry.

\section{Acknowledgement}

This work is partially supported by1. the National Philosophy and Social Science Foundation as an important program, the Assurance Mechanism of Agricultural Product Quality and Safety based on Multi-agent Coordination(No. 15AGL014). It is also partially supported by 2.the Important Research and Development Program of Shandong Province in 2016, Key Issues and System Implementation of Early Warning for Agricultural Product Quality Safety under the Trend of Intelligent Agriculture (No. 2016GSF120013). 3. The Technology System Innovation Plan of Chinese Herbal Medicine Industry of Shandong Province on the 13th Five-Year Plan, The authors would like to acknowledge the referees for their valuable comments and suggestions which helped to improve the presentation of this paper.

\section{Reference}

[1] Jiansheng Liu. Countermeasures for the development of industrialization of Chinese herbal medicine [J]. Agricultural development and equipment, 2016.08.

[2] Jiehua Lv, Guan Lin. Chinese herbal medicine industry chain under review of Linxia [J]. Forestry economics, 2015.07 (82-85).

[3] Jian Ouyang. Use the whole industry chain to do "green ecological long medicine" [J], Gansu agriculture, 2017.03 (8-11). 